Review

\title{
Th17 Cells as Potential Probiotic Therapeutic Targets in Inflammatory Bowel Diseases
}

\author{
Eddy Owaga ${ }^{1}$, Rong-Hong Hsieh ${ }^{2}$, Beatrice Mugendi ${ }^{1}$, Sakhile Masuku ${ }^{3}$, Chun-Kuang Shih ${ }^{2}$ \\ and Jung-Su Chang ${ }^{2, *}$
}

1 Institute of Food and Bioresources Technology, Dedan Kimathi University of Technology, 10100 Nyeri, Kenya; E-Mails: eowaga@yahoo.co.uk (E.O.); beatrice.mugendi@dkut.ac.ke (B.M.)

2 School of Nutrition and Health Sciences, College of Public Health and Nutrition, Taipei Medical University, $250 \mathrm{Wu}$-Xing Street, 110 Taipei, Taiwan; E-Mails: hsiehrh@tmu.edu.tw (R.-H.H.); ckshih@tmu.edu.tw (C.-K.S.)

3 Community Health Nursing Department, Faculty of Health Sciences, University of Swaziland, H100 Mbabane, Swaziland; E-Mail: SMasuku@uniswa.sz

* Author to whom correspondence should be addressed; E-Mail: susanchang@tmu.edu.tw; Tel.: +886-2-2736-1661 (ext. 6542); Fax: +886-2-2737-3112.

Academic Editor: Kamal D. Moudgil

Received: 19 June 2015 / Accepted: 25 August 2015 / Published: 1 September 2015

\begin{abstract}
Inflammatory bowel diseases (IBD) are characterized by wasting and chronic intestinal inflammation triggered by various cytokine-mediated pathways. In recent years, it was shown that $\mathrm{T}$ helper 17 (Th17) cells are involved in the pathogenesis of IBD, which makes them an attractive therapeutic target. Th17 cells preferentially produce interleukin (IL)-17A-F as signature cytokines. The role of the interplay between host genetics and intestinal microbiota in the pathogenesis of IBD was demonstrated. Probiotics are live microorganisms that when orally ingested in adequate amounts, confer a health benefit to the host by modulating the enteric flora or by stimulating the local immune system. Several studies indicated the effectiveness of probiotics in preventing and treating IBD (ulcerative colitis, and Crohn's disease). Furthermore, there is mounting evidence of probiotics selectively targeting the Th17 lineage in the prevention and management of inflammatory and autoimmune diseases such as IBD. This review highlights critical roles of Th17 cells in the pathogenesis of IBD and the rationale for using probiotics as a novel therapeutic approach for IBD through manipulation of Th17 cells. The potential molecular mechanisms by which probiotics modulate Th17 cells differentiation and production are also discussed.
\end{abstract}


Keywords: Th17; IL-17; probiotics; inflammatory bowel diseases; inflammation

\section{Introduction}

Inflammatory bowel diseases (IBD) are characterized by chronic inflammation of the gastrointestinal (GI) tract. The global prevalence of IBD ranges $12 \%-20 \%$ [1]. The two major types of IBD include Crohn's disease (CD, inflammation of any section of gastrointestinal tract), and ulcerative colitis (UC, inflammation of the lining of the large intestine) [2]. In addition, pouchitis describes the inflammation of the intestinal wall of the ileal reservoir, which is associated with colectomy and restorative surgery [2]. The pathogenesis of IBD is mainly linked to uncontrolled mucosal inflammation due to an abnormal immune response against luminal antigens and microbiota [3]. This response is mainly triggered by a breakdown in the intestinal homeostasis among the microbiota, intestinal epithelial cells, and resident immune cells [4]. Innate and adaptive immune responses are important factors in the development of IBD [5]. In the early phase of inflammation, innate immune cells, including mast cells, neutrophils, macrophages and dendritic cells, located in the intestine are recruited in response to antigens and commensal bacteria. Subsequently, the persistent inflammation arising from the released proinflammatory innate immune cells results in activation of an adaptive immune response [4]. Both innate and adaptive immune responses result in chronic tissue injury and epithelial damage related symptoms in IBD patients $[1,4]$.

Numerous previous studies showed that $\mathrm{CD}^{+} \mathrm{T}$ helper (Th)1 and $\mathrm{Th} 2$ cells are essential in the pathogenesis of IBD [6,7]. CD and UC are widely associated with Th1 cytokines (tumor necrosis factor (TNF)- $\alpha$; interferon (IFN)- $\gamma$; interleukin (IL)-12) and Th2 cytokines (IL-5 and IL-13) in the mucosa [4,5]. Nevertheless, despite extensive research on the roles of Th1 and Th2 cytokines, knowledge of factors causing IBD remains incomplete. Recently, a new distinct subset of Th17 cells capable of producing IL-17 was associated with the IBD pathogenesis due to their proinflammatory roles in the mucosal immune response [4,6]. Secreted IL-17 exists in six isoforms: IL-17A-F [4,6]. In addition to the IL-17 isoforms, Th17 cells also differentiate and secrete other proinflammatory cytokines such as IL-6, IL-1 $\beta$, TNF- $\alpha$, IL-12, tumor growth factor (TGF)- $\beta$, IL-23, IL-21, IL-22, and IL-26 during the IBD pathogenesis [6].

Several human studies demonstrated the pathogenic role of Th17 in IBD. According to Sartor [8], IL-17 messenger (m)RNA is highly expressed in inflamed mucosa obtained from both UC and CD patients. In other comparative studies, the number of Th17 cells and IL-17 expression were markedly enhanced in the inflamed gut of CD and UC patients $[9,10]$. In addition, elevated levels of IL-17 were reported in intestinal tissues and serum of IBD patients [11]. Further evidence of the role of Th17 in IBD development is the strong correlation between the disease severity and IL-17 levels in speripheral blood mononuclear cells from UC patients [12]. Moreover, in a cohort study of subjects with CD and UC, patients with IBD showed a remarkably higher prevalence of circulating IL-17 than did the control group [10].

Several animal studies also showed etiological implications of IL-17A in IBD pathogenesis. Ito et al. [13] observed diminished manifestations of colitis in mice with a deficiency of the IL-17A gene after administration of dextran sodium sulfate (DSS). In addition, Zhang et al. [14] also reported that IL-17R-knockout mouse were significantly protected against colonic inflammation in a model of 
trinitrobenzenesulfonic acid (TNBS)-induced colitis. Taken together, these observations imply that Th17 cytokines are strongly linked to the pathogenesis of IBD and hence are an attractive therapeutic target for IBD prevention and management.

The primary aim of this review is to highlight critical roles of Th17 cells in the pathogenesis of IBD and the rationale for using probiotics as a novel therapeutic approach through manipulation of Th17 cells in IBD. Specifically, this review examines the modulatory role of probiotics on Th17 in IBD in vitro, and in animal and human studies, and provides insights into the potential underlying molecular mechanisms of probiotic modulation of Th17 towards alleviating IBD.

\section{Role of Th17 Cells in the Gut Immune Homeostasis}

Roles of Th17 cells in intestinal pathology and homeostasis still remain poorly understood. Nevertheless, as outlined below, some of the suggested roles in gut-related tissues include; (i) promotion of microbial defense; (ii) modulation of $\mathrm{T}$ cell differentiation and cellular production of inflammatory mediators [6]; and (iii) modulation of neutrophil migration and function.

Although controversial, it has been suggested that Th17 cells may confer a protective effect on the GI tract under steady-state homeostatic regulation, but initiate an uncontrolled pathogenic immune response during dysbiosis [5,15-18]. Th17 cells are particularly believed to clear fungal and extracellular bacterial infections that are not efficiently cleared by Th1- and Th2-type immunity [5,19]. IL-17A production has been reported upon infection with Staphylococcus aureus, Clostridium rodentium, Klebsiella pneumonia, Mycobacterium tuberculosis, Leuconostoc monocytogenes, Salmonellae typhimurium, and Pneumocystic carinii [20]. IL-17A was also shown to fortify tight junction formation between epithelial cells by inducing expressions of claudins and by stimulating mucin production by intestinal epithelial cells, thereby increasing the integrity of the intestinal barrier [21,22]. In humans, the suppression of Th17 production due to defective STAT3 gene mutation has been linked to hyper-immunoglobulin E syndrome (HIES). HIES is characterized by abnormal susceptibility to S. aureus, S. pneumonia, and C. albicans infections [20]. In animal studies, a protective role was suggested for IL-17A in a T-cell transfer model of colitis [23,24]. In agreement with this observation, treatment of mice with an anti-IL-17 neutralizing antibody enhanced the severity of DSS-induced colitis [22]. It was also revealed that murine DSS-induced colitis was worsened in IL-17A-knockout mice but was substantially improved in IL-17F-knockout mice [25]. These contrasting results on the pathogenic and protectivde roles of Th17 are, at least partly, attributed to differences in ligand affinity, downstream signalling cascades, and receptor tissue distribution [4] and other factors that influence the action of Th17 and may be present in the local environment [5].

Notably, of critical relevance to this review is the pathogenic role of Th17 in IBD. Th17 cells are involved in the priming and differentiation of proinflammatory cytokines in the IBD pathogenesis. After activation, Th17 cells secrete various isoforms of cytokine IL-17 (IL-17A-F), which eventually stimulate intestinal endothelial cells, myofibroblasts, and epithelial cells to produce other proinflammatory mediators [14,23]. Furthermore, several studies demonstrated that Th17 cells can directly induce the secretion of several proinflammatory effector molecules which mediate tissue infiltration and tissue destruction, including proinflammatory cytokines (IL-6, IL-1 $\beta$, TNF- $\alpha$, IL-12, TGF- $\beta$, IL-23, IL-21, IL-22, and IL-26), chemokines, and matrix metalloproteases [4-6,26]. The imbalance in the microbiota profile 
in the gut, can transform the steady "peace" state immune status in healthy individuals to activated "war" state characterized by rapid expansion of Th17 cells [4]. For instance, proliferation of pathogens provides Toll-like receptor (TLR) ligands that activate dendritic cells to produce IL-6 and TGF- $\beta$, which subsequently promote differentiation of Th17 cells. Uncontrolled production of Th17 cells can lead to dysregulated production of pro-inflammatory cytokines and chronic inflammation, eventually contributing to tissue damage in IBD [20]. For this reason, modulation of the differentiation and function of Th17 cells is currently viewed as a potentially feasible therapeutic target for intestinal inflammatory conditions experienced in IBD.

Emerging data suggests IL-17 can influence neutrophil migration and function during inflammation. Grifin et al. [27] found that IL-17 enhanced expression of neutrophilic chemokines CXCL1, CXCL2, and CXCL5 through endothelial activation. Taylor et al. [28] also reported human and mouse neutrophils that were produced and responded to IL-17A. In animal models, endogenous IL-17 mediated the recruitment of neutrophils in mouse airways upon endotoxin exposure [29].

\section{Risk Factors for the Development of IBD}

The pathogenesis of IBD has been associated with interaction of multiple factors, namely: (i) genetic susceptibility factors; (ii) host immunity homeostasis; (iii) integrity of the intestinal epithelial cells; and (iv) environmental factors, such as microbiota [30-32].

\subsection{Genetic Susceptibility Factors}

Generally, IBD arise from a disruption of mucosal immune homeostasis in genetically susceptible individuals [5,33]. A recent genome-wide study on the role of Th17 cells in the pathogenesis of IBD showed that genes involved in Th17 differentiation are associated with a susceptibility to IBD [2,34]. On the other hand, it was found that a mutation of the gene encoding the IL-23 receptor (IL-23R) is strongly associated with IBD [35]. Siakavellas and Bamias [36] also identified several CD-associated polymorphisms in genes that encode for proteins of the IL-23/Th17 pathway. Other genome-wide association studies also revealed polymorphisms in additional genes that are linked to a risk of IBD pathogenesis. Some of the genes implicated in the onset of both CD and UC are essential for intestinal homeostasis, including T cell regulation (TNFSF8, IL12B, IL23R, PRDM1, and ICOSLG), immune tolerance (IL10 and CREM), innate mucosal defense (CARD9 and REL), restitution (REL, PTGER4, and NKX2-3), paneth cells (XBP1), and immune cell recruitment (MST1) [2]. Bogoaert et al. [37] studied differential mucosal expression of genes involved in differentiation (IL-6, IL-1 $\beta, T G F-\beta, I L-23 A$ and STAT3) and recruitment of Th17 cells (CCR6 and CCL20) and observed enhanced expression of these genes in ileal and colonic samples from UC and CD patients. Seiderer et al. [38] reported increased colonic IL17F gene expression in active $\mathrm{CD}$.

\subsection{Host Immunity Homeostasis}

The gastrointestinal tract regulates immune responses of the immune system against pathogens and commensals, thereby maintaining homeostasis of the human gut [4]. It is generally accepted that chronic intestinal inflammation and damage arise from excessive and uncontrolled mucosal immune responses 
(particularly by $\mathrm{CD}^{+} \mathrm{T}$ cells) against commensal microbes in the gut in individuals with a genetic susceptibility $[6,18]$. Traditionally, CD and UC have been associated with Th1 cytokines (TNF $\alpha$, IFN $\gamma$, IL-12), and Th2 cytokines (IL-5, IL-13), respectively [5]. In addition, inflammation in UC is continuous in the colon whereas inflammation in $\mathrm{CD}$ may lead to inflamed sections mixed with healthy parts of intestine [4].

\subsection{Integrity of Intestinal Epithelial Cells (IECs)}

IECs are important components of the intestinal barrier between mucosal immune cells and luminal tissues, which are crucial in maintaining the immune homeostasis of the gut, by physically separating commensal bacteria and mucosal immune cells [3]. In addition, IECs integrate microbial signals into the mucosal immune system through pattern-recognition receptors [30]. Using a specific Ripk1 mice model, it was established that mice developed severe intestinal inflammation due to IEC death and subsequent dysfunction of the intestinal barrier function [39]. To further support the role of IECs in the pathogenesis of IBD. Dotan et al. [34] found that IECs from IBD patients excessively induced $\mathrm{CD}^{+} \mathrm{T}$ cell proliferation and produced interferon (IFN) $\gamma$ due to disturbed intestinal immune homeostasis. Overall, those studies revealed that alterations of the integrity of IECs drive chronic intestinal inflammation, and hence controlling IECs is essential to prevent uncontrolled mucosal inflammation.

\subsection{Environmental Factors such as Microbiota}

The role of the gut microbiota in the pathogenesis of human IBD was highlighted by several authors $[4,40]$. Interactions of microbes with mucosal immune cells in the GI tract are important in priming and regulating immunity [41]. Thus, increased evidence showed that IBD primarily represent an imbalance of the intestinal microbiota and a malfunction of tolerance to commensal microbiota [42]. Changes in the commensal bacterial floral composition within the GI tract are responsible for altering immune responses from a steady state to activated, aggressive, and damaging immune responses characterized by expansion of Th17 cells [31]. There is mounting evidence that normal microbiota such as Lactobacillus spp. and Bifidobacteria spp. in the GI tract are significantly reduced in patients with IBD $[43,44]$ but instead are associated with susceptibility to pathogens such as Clostridium difficile [45], Actinobacteria and Proteobacteria [46], and Streptococcus spp. [47]. According to Gálvez [4] pathogen-infected epithelial cells provide Toll-like receptor (TLR) ligands, leading to secretion of IL-6 and TGF- $\beta$ from dendritic cells, hence activating Th17 differentiation in IBD.

\section{Potential Therapeutic Roles of Probiotics in IBD}

In the recent past, there was intense research on developing new therapeutics, such as probiotics, which can reduce intestinal inflammation and restore a balance to the GI tract microbiota in IBD. Probiotics are defined as living microorganisms which when consumed in adequate amounts confer health benefits to the host beyond inherent general nutrition $[17,48]$. However, a considerable number of recent studies indicated that beneficial effects can also be achieved by heat-inactivated probiotics, isolated bacterial DNA, or probiotic-cultured media $[49,50]$. Lactobacilli and bifidobacteria are 
predominantly present in the ileum and colon, and hence both have been widely studied for preventing and managing IBD [17,31,51,52].

The efficacy of probiotics in IBD has been examined in various animal models and clinical studies, and respective outcomes were reviewed by several authors [1,3,31,41,51,53,54]. Despite a few cases of conflicting results, probiotics have generally successfully been demonstrated to have some efficacy in alleviating inflammation in human IBD and murine colitis models. Several clinical studies indicated the effectiveness of specific probiotic strains towards reduction of UC severity. These include $L$. casei subsp. rhamnosus [55]; Bifidobacteria breve, B. bifidum, L. acidophilus [56]; VSL\#3 (mixture of L. plantarum, acidophilus, L. casei, L. delbruecki subsp. bulgaricus, B. longum, B. infantis, B. breve and Streptococcus thermophilus) [57]. In contrast, other studies have shown no significant benefit of E. coli Nissle 1917 [58] and a mixture of L. salivarius and B. infantis [45]. Several clinical trials have also demonstrated efficacy of probiotics in alleviating inflammation in CD. These include VSL\#3 [59], and S. boulardii [60]. However, other clinical trials have reported no effect of some probiotics in preventing CD such as L. rhamnosus LGG [61-64], and L. johnsonii LA1 [65,66]. Some clinical studies have shown beneficial effect of probiotics on pouchitis including VSL\#3 [67,68] and L. rhamnosus LGG [69]. In contrast, other comparative studies have shown no significant effect of VSL\#3 [70] and L. rhamnosus LGG [71] on pouchitis compared to respective controls. Several studies conducted using murine models have demonstrated the effectiveness of probiotics in alleviating TNBS- or DSS-induced colitis. These include L. fermentum [50,72-74], L. acidophilus [75], B. longum subsp. infantis JCM 1222 [32] and S. thermophilus ST28 [17].

As suggested by various authors [31,76], inconsistencies in outcomes of some clinical trials of IBD can be explained in several ways: (i) marked heterogeneity between IBD trials with regard to choices of probiotic and their dose, the trial design, and outcome measures evaluated; (ii) variations in the populations studied, with some trials enrolling patients with active disease; (iii) most studies having enrolled small numbers of patients, which limits the statistical power and may account for the high placebo response rates reported in IBD clinical trials; and (iv) most studies not indicating details of the patients' diets, which may potentially influence the efficacy of the probiotics.

\section{Mechanisms Underlying Probiotic Anti-Inflammatory Effects on IBD Pathogenesis}

Although it is apparent from animal and human studies that probiotics may confer relief of IBD symptoms to the host upon consumption, the exact mechanisms by which probiotics impart protective effects are still not fully understood. As briefly outlined below, multiple broad mechanisms have been suggested including: (i) displacement and suppression of the growth of pathogens [17]; (ii) improved epithelial barrier function [32,50]; and (iii) immunomodulation of Th1, Th2, regulatory T cell, and Th17 cell production [17].

\subsection{Displacement and Suppression of Growth of Pathogens}

Probiotics are believed to rapidly colonize the gastrointestinal tract hence competitively inhibit pro-adherence of pro-inflammatory pathogens to the IEC [31,32]. Several invasive pathogenic bacteria such as $E$. coli and $C$. difficile are associated with IBD development [77,78]. A number of human studies have demonstrated that after administration of VSL\#3 probiotic mixture, the relief of the inflammation 
conditions in IBD patients are accompanied by increased colonization of the gastrointestinal tract [64,79]. Administration of L. acidophilus also led to increased lactobacilli and bifidobacteria profile but reduced Staphylococcus aureus population in the distal colon section [75]. In murine models, some authors reported that L. fermentum ACA-DC 179 [73] and Duolac Gold (mixture of lactobacilli bacteria) [48] inhibited the growth of Salmonella and C. difficile, respectively.

\subsection{Improved Epithelial Barrier Function}

Several studies have demonstrated that the metabolites produced by bacteria may interact directly with gut epithelial cells to enhance mucosal barrier function [80]. Peran et al. [50] reported that heat-killed L. brevis SBC8803 ameliorates intestinal injury in a murine model of colitis by enhancing the intestinal barrier function. The exact mechanisms underlying improved epithelial barrier function are yet to be elucidated.

\subsection{Immunomodulation of Th1, Th2, Regulatory T Cells, and Th17 Cells Production}

In the intestinal tract, immunocytes such as $\mathrm{M}$ cells and dendritic cells are constantly responding to intestinal bacteria, an event referred to as microbe-host "cross-talk" [40,51,81]. Consequently, upon consumption, probiotic bacteria or cell wall components are internalized by $\mathrm{M}$ cells to interact with dendritic cells, which further influence the polarization of T-cell responses (Th1, Th2, or regulatory T cells) [40]. Therefore, it is generally recognized that the anti-inflammatory effect of probiotics in IBD is linked to the down-regulation of pro-inflammatory IL-17 cytokine production and upstream Th-17 secreted cytokines (IL-23, TGF- $\beta$ ) and downstream Th-17 secreted cytokines (IL-1 $\beta$, IL-6, TNF- $\alpha$, IFN $\gamma$, IL-12).

\subsubsection{Immunomodulation of IL-17 and IL-23 Cytokines}

Several animal experiments and clinical trials have demonstrated that anti-inflammatory effects of probiotics in IBD might be a consequence of the down regulation of pro-inflammatory IL-17 production $[17,26]$. Various studies on colitis-induced animal models have revealed several probiotics that can down regulate IL-17 production as well as simultaneously alleviate colitis. These probiotics include B. breve [33], B. longum [32], L. acidophilus [6], B. longum subsp. infantis [26], S. thermophilus ST28 [82], and L. gasseri A5 [33]. The feasibility of the proposed mechanism of probiotic inhibition of IL-17 target is supported by a comparative study showing 4SC-101 [83] and Vidofludimus [84], both novel immunosuppressive drugs, inhibit IL-17 and attenuates chronic colitis in mice. IL-23 is responsible for expansion, stabilization and conditioning of Th17 hence its key role in the activation of inflammation in IBD [6]. The IL-23/17 axis is widely regarded as a hallmark of IBD and is therefore an attractive probiotic therapeutic target in IBD prevention and management $[18,33,85]$. Indeed, Ghadimi et al. [33] investigated the effect of B. breve and L. rhamnosus GG on the expression of IL-23 on intestinal cells and found diminished liposaccharide-induced expression of IL-23.

\subsubsection{Immunomodulation of TGF- $\beta$, IL- $1 \beta$ and IL- 6 Cytokines}

TGF- $\beta$ regulates Th17 differentiation by acti $\beta$ ating transcription factor signal transducer for transcriptor 3 (STAT3) [86]. Kumar et al. found reduced expression and production of TGF- $\beta$ in colitis 
mouse models after administration of probiotic mixture comprising four lactobacilli and eight Bifidobacterium spp. [87]. IL-1 $\beta$ is critical for induction differentiation and development of Th17 [88]. Reduced expression of IL- $\beta$ in the colon have been found in colitis mouse models after oral administration of L. brevis SBC 8803 [89], and lactobacilli mixture [90]. IL-6 has been shown to initiate Th17 differentiation in IBD pathogenesis. Several studies have shown reduced levels of IL-6 expression and production as well as parallel relief of intestinal damage in DSS- and TNBS-induced colitis mice models after administration of probiotics. These include L. fermentum CECT 5716 [74], Duolac Gold (mixture of seven species of bifidobacteria, lactobacilli bacteria and S. thermophilus) [48], and lactobacilli mixture [90].

\subsubsection{Immunomodulation of TNF- $\alpha$ and IFN $\gamma$ Cytokines}

TNF- $\alpha$ is among the pro-inflammatory cytokines secreted downstream of Th17 cells differentiation during IBD pathogenesis. Several authors have reported the ability of several probiotics to down regulate the expression and production of TNF- $\alpha$ in DSS- and TNBS-induced colitis mouse, namely L. brevis SBC 8803 [89], mixture of lactobacilli and bifidobacteria [91], L. fermentum [50], B. lactis [92], L. salivarius subsp. salivaris [93] and probiotic mixture of four lactobacilli spp. and eight Bifidobacterium spp. [87]. IFN $\gamma$ is secreted downstream of Th17 activation hence contribute to inflammation in IBD. Several studies in mouse models of induced colitis have shown probiotics can inhibit expression and production of IFN $\gamma$. These include lactobacilli and bifidobacteria mixture [91], and lactobacilli mixture [90].

\subsubsection{Immunomodulation of IL-12 and IL-10 Cytokines}

IL-12 production also occurs downstream of Th17 activation in IBD. Some studies have shown probiotic s such as L. brevis SBC 8803 [89], and lactobacilli and bifidobacterium spp. mixture [91] can inhibit expression and production of IL-12 in colitis mouse models. The down-regulation of Th17 cells production by probiotics also has been associated with the up-regulation of anti-inflammatory cytokines such as IL-10, which are vital in the maintenance of the immune balance. Several studies on anti-inflammatory potential of probiotics have shown increased expression and production levels of IL-10 after administration to colitis mouse models. These include B. longum subsp. infantis JCM 1222T [26], L. salivarius Ls 33, E. coli Nissle 1917 [94], L. plantarum [95], L. rhamnosus GG, S. thermophilus, B. animalis subsp. lactis, B. breve [96], VSL\#3 mixture [97], lactobacilli and bifidobacteria mixture [91] and L. fermentum [73]. The IL-10/IL-12 ratio is relatively low in IBD patients and studies have shown that some probiotics such as VSL \#3 mixture have a potential of inducing IL-10 production in IBD subjects [17]. In addition, high IL-10/IL-12 ratio, a common indicator of the probiotic anti-inflammatory effect potential, was induced after administration of B. longum subsp. infantis [98].

Overall, the discrepancies on the beneficial effects between the various probiotic species and strains, have largely been attributed to the differences in the cell wall structure of the probiotic strains especially the microbe-associated molecular patterns such as lipoteichoic acid, peptidoglycan, and non-methylated CpG motifs [75]. Due to the limited number of human studies, most of the hypotheses on IBD pathogenesis have been assessed using mouse models, which have led to improved understanding of the mechanisms underlying pathogenesis of IBD [99]. However, it is important to interpret the mouse model 
results with caution because of the emerging concerns on the variations in the origin and plasticity of human and murine Th17 cells [100].

\section{Potential Molecular Mechanisms for Th17 Suppression by Probiotics}

The mechanisms by which probiotics modulate Th17 cell differentiation and production in the gut still remain to be fully elucidated. As briefly described below, some of the emerging potential mechanisms include: (i) inhibition of co-stimulatory molecules (CD40 and CD80) in IECs; (ii) downregulation of Th17 cell transcription factors retinoic-acid-related orphan receptor (ROR) $\gamma$ t, STAT3, and nuclear factor

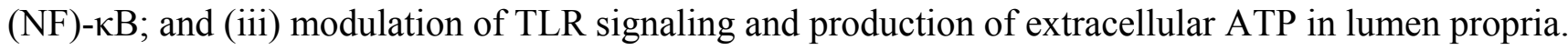

\subsection{Inhibition of Co-Stimulatory Molecules in IEC (CD40, CD80)}

As antigen-presenting cells, inflamed IECs could function by expressing higher levels of costimulatory molecules, which cause abnormal activation of T cells [101,102]. Indeed, using mouse intestinal epithelial cell line Colon-26 cells, it was demonstrated that inflamed IECs induce IL-17A response via co-stimulatory molecules CD80/CD86 and CD40 [103]. Thus, these molecules are considered as important IBD-causing factors and therefore potential probiotic therapeutic target for IBD. In this regard, B. longum subsp. infantis JCM 1222 has been shown to directly act on IECs to suppress CD80 and CD40 expression, and subsequently resulting in the suppression of mRNA and protein expression levels of IL-17A and alleviation of colitis [32]. In another comparative study, B. breve and L. rhamnosus GG suppressed the liposaccharide-induced mRNA expression of CD40 in an in vitro model of the human intestinal HT-29/B6 or T84 cells mucosal immune system [33]. As a whole, these modulatory effects give an insight on the novel therapeutic role of probiotics in alleviating IBD via suppression of the costimulatory molecules in IECs such as CD80/CD86 and CD40, which are vital in activating expression of IL-17A during IBD pathogenesis.

\subsection{Down-Regulation of Th17 Cell Transcription Factors ROR $\gamma t$, STAT3, and NF- $k B$}

Retinoic-acid-related orphan receptors (ROR $\gamma \mathrm{t}$ ), has been identified as the transcription factor for Th17 cells [4,5]. Indeed, Ivanov [104] did not observe Th17 in ROR $\gamma$ t-deficient mice, but the production of Th17 was induced upon transduction of naive T cells with a ROR $\gamma t$-encoding retrovirus, hence demonstrating the integral role of ROR $\gamma \mathrm{t}$ in Th17 cells differentiation. Thus, it has been proposed that ROR $\gamma \mathrm{t}$ could be potential probiotic therapeutic target towards inhibition of Th17 cells in IBD [10]. Subsequently, upon oral administration of B. longum subsp. infantis, [32] observed suppressed production of Th17-cytokines (IL-17A), which was parallel to down-regulated expression of ROR $\gamma \mathrm{t}$.

The NF-KB and STAT3 signaling pathway is critical in promoting expression of pro-inflammatory Th-17 cell secreted cytokines, leading to intestinal inflammation in IBD pathogenesis $[4,18,105]$. In addition, transcription factor STAT3 has been reported to regulate the expression of the transcription factor ROR $\gamma$ t, which is critical in Th17 differentiation and development [6]. To demonstrate the role of STAT3 in Th17 development, Galvez [4] reported that STAT3 overexpression promoted Th17, but the process was impaired in mice with STAT3-deficient cells. Furthermore, genome wide association studies have revealed polymorphism in Th-17 related genes such as STAT3 in IBD patients [2,33]. 
Therefore modulation of both the NF-kB and STAT3 mediated transcriptions of IBD — Causing Th-17 cell factors is viewed as a novel probiotic therapeutic target in prevention and management of IBD [18,33]. Indeed, Ghadimi et al. [106] reported that B. longum suppressed the activation of epithelial NF-KB via Toll-like receptor 9 in a colitis mouse model. Comparative studies B. breve and L. rhamnosus GG have also shown reduced nuclear translocation of NF-кB in human intestinal HT-29/B6 T84 cells [33]. Other probiotic bacteria that have exhibited suppressed activation of NF-kB in colitis mouse models include: L. plantarum HY115 and L. brevis HY7401 mixture [90], B. breve, S. thermophilus, and B. bifidum mixture [107]. In addition, a clinical trial of IBD patients showed Bifidobacterium supplements reduced the expression of the pro-inflammatory transcription factor NF-KB. Regarding probiotic effect on STAT3 transcription, a study by Chen et al. [6] demonstrated L. acidophilus suppressed IL-17 production through down regulation of mRNA expression and phosphorylation of STAT3 [6]. Further, to support the novel role of NF-KB and STAT3 as potential therapeutic targets in prevention and treatment of IBD, Fitzpatrick [18] reported that Vidofludimus, an antibody drug, could inhibit IL-17 secretions in activated splenocytes by inhibiting STAT3 and NF-кB signaling pathways.

\subsection{Modulation of TLR Signaling and Production of Extracellular ATP in Lumen Propria}

Recent research advances by Atarashi et al. [108], show that production of Th17 cells in the intestinal lamina propria is, at least partly, regulated by the commensal bacteria. The two main hypothesized mechanisms include modulation of TLR and extracellular ATP [109]. Commensal bacteria release TLR ligands which stimulate lamina propria dendritic cells leading to differentiation of Th17 cells. In addition, commensal bacteria release extracellular ATP, which activate the dendritic cells to induce production of IL- 6 and TGF- $\beta$ cytokines leading to differentiation of Th17 cells.

\section{Conclusions}

More data from animal experiments and clinical trials support the potential therapeutic role of probiotics in IBD. Generally, the efficacy of probiotics in IBD is more evident in UC than in CD. Increasing evidence shows a new distinct subset of T helper cells (Th17), that are capable of producing IL-17 cytokines, plays key pathogenic roles in IBD due to their proinflammatory role in the mucosal immune response. The anti-inflammatory effects of probiotics in IBD might be a consequence of downregulation of IL-17 production and related proinflammatory Th17-secreted cytokines such as IL-23, TGF- $\beta$, IL-1 $\beta$, IL-6, TNF- $\alpha$, IFN- $\gamma$, and IL-12. Some of the emerging potential molecular mechanisms for the probiotics modulation of Th17 cell differentiation and production in the gut include inhibition of co-stimulatory molecules (CD40 and CD80) in IECs; downregulation of the Th17 cell transcription factors, ROR $\gamma \mathrm{t}$, STAT3, and NF-kB; and modulation of TLR signaling and production of extracellular ATP in lumen propria. Further studies are required to elucidate which bioactive components from probiotic bacteria are responsible for the anti-inflammatory effects in IBD. Moreover, some critical differences appear to exist with regard to the pathogenic roles of various IL-17 isoforms in IBD; therefore, further studies should explore how probiotics can selectively alter expressions and production of specific IL-17 isoforms. It is anticipated that novel anti-inflammatory mechanisms hereby highlighted will improve our knowledge of how probiotics alleviate IBD pathogenesis and thus lead to the efficient 
identification and selection of beneficial probiotic strains with the goals of preventing and managing IBD symptoms.

\section{Acknowledgments}

Jung-Su Chang was supported by grants from Taipei Medical University Hospital (103TMU-TMUH-11 and 104TMU-TMUH-18) and the Ministry of Science and Technology, Taiwan (MOST 103-2320-B-038015 and MOST 104-2311-B-038-005).

\section{Author Contributions}

Rong-Hong Hsieh and Jung-Su Change conceived the idea and scope of the paper, Eddy Owaga drafted the initial manuscript and critically revised the manuscript, Beatrice Mugendi, Sakhile Masuku and Chun-Kuang Shih screened the literature and checked the overall coherence of the paper.

\section{Conflicts of Interest}

The authors declare no conflict of interest.

\section{References}

1. Dai, C.; Zheng, C.-Q.; Jiang, M.; Ma, X.Y.; Jiang, L.J. Probiotics and irritable bowel syndrome. World J. Gastroenterol. 2013, 19, 5973-5980.

2. Khor, B.; Gardet, A.; Xavier, R.J. Genetics and pathogenesis of inflammatory bowel disease. Nature 2011, 474, 307-317.

3. Sheil, B.; Shanahan, F.; O’Mahony, L. Probiotic effects on inflammatory bowel disease. J. Nutr. 2007, 137, 819S-824S.

4. Gálvez, J. Role of Th17 cells in the pathogenesis of human IBD. ISRN Inflamm. 2014, 2014, 928461.

5. Liu, Z.-J.; Yadav, P.K.; Su, J.-L.; Wang, J.S.; Fei, K. Potential role of Th17 cells in the pathogenesis of inflammatory bowel disease. J. Gastroenterol. 2009, 15, 5784-5788.

6. Chen, L.; Zou, Y.; Peng, J.; Lu, F.; Yin, Y.; Li, F.; Yang, J. Lactobacillus acidophilus suppresses colitis-associated activation of the IL-23/Th17 axis. J. Immunol. Res. 2015, 2015, 909514.

7. Sarra, M.; Pallone, F.; MacDonald, T.T.; Monteleone, G. IL-23/IL-17 axis in IBD. Inflamm. Bowel Dis. 2010, 16, 1808-1813.

8. Sartor, R.B. Mechanisms of disease: Pathogenesis of Crohn's disease and ulcerative colitis. Nat. Clin. Pract. Gastroenterol. Hepatol. 2006, 3, 390-407.

9. Hölttä, V.; Klemetti, P.; Salo, H.M.; Koivusalo, A.; Pakarinen, M.; Westerholm-Ormio, M.; Kolho, K.L.; Vaarala, O. Interleukin-17 immunity in paediatric Crohn disease and ulcerative colitis. J. Pediatr. Gastroenterol. Nutr. 2013, 57, 287-292. 
10. Ueno, A.; Jijon, H.; Chan, R.; Ford, K.; Hirota, C.; Kaplan, G.G.; Beck, P.L.; Iacucci, M.; Fort, G.M.; Barkema, H.W.; et al. Increased prevalence of circulating novel IL-17 secreting Foxp3 expressing $\mathrm{CD}^{+} \mathrm{T}$ cells and defective suppressive function of circulating Foxp $3^{+}$regulatory cells support plasticity between Th17 and regulatory $\mathrm{T}$ cells in inflammatory bowel disease patients. Inflamm. Bowel Dis. 2013, 19, 2522-2534.

11. Sahin, A.; Calhan, T.; Cengiz, M.; Kahraman. R.; Aydin, K.; Ozdil, K.; Korachi, M.; Sokmen, H.M. Serum interleukin 17 levels in patients with Crohn's disease: Real life data. Dis. Markers 2014, 2014,690853

12. Raza, A.; Shata, M. Letter: Pathogenicity of Th17 cells may differ in ulcerative colitis compared with Crohn's disease. Aliment. Pharmacol. Ther. 2012, 36, 204-204.

13. Ito, R.; Kita, M.; Shin-Ya, M.; Kishida, T.; Urano, A.; Takada, R.; Sakagami, J.; Imanishi, J.; Iwakura, Y.; Okanoue, T.; et al. Involvement of IL-17A in the pathogenesis of DSS-induced colitis in mice. Biochem. Biophys. Res. Commun. 2008, 377, 12-16.

14. Zhang, Z.; Zheng, M.; Bindas, J.; Schwarzenberger, P.; Kolls, J.K. Critical role of IL-17 receptor signaling in acute TNBS-induced colitis. Inflamm. Bowel Dis. 2006, 12, 382-388.

15. Bedoya, S.K.; Lam, B.; Lau, K.; Larkin, J., III. Th17 cells in immunity and autoimmunity. Clin. Dev. Immunol. 2013, 2013, 986789.

16. Awasthi, A.; Kuchroo, V.K. Th17 cells: From precursors to players in inflammation and infection. Int. Immunol. 2009, 21, 489-498.

17. Tanabe, S. The effect of probiotics and gut microbiota on Th17 cells. Int. Rev. Immunol. 2013, 32, 511-525.

18. Fitzpatrick, L.R. Novel pharmacological approaches for inflammatory bowel disease: Targeting key intracellular pathways and the IL-23/IL-17 axis. Int. J. Inflamm. 2012, 2012, 389404.

19. Annunziato, F.; Cosmi, L.; Liotta, F.; Maggi, E.; Romagnani, S. The phenotype of human Th17 cells and their precursors, the cytokines that mediate their differentiation and the role of Th17 cells in inflammation. Int. Immunol. 2008, 20, 1361-1368.

20. Jin W.; Dong, C. IL-17 cytokines in immunity and inflammation. Emerg. Microbes Infect. 2013, 2, e60.

21. Kinugasa, T.; Sakaguchi, T.; Gu, X.; Reinecker, H.C. Claudins regulate the intestinal barrier in response to immune mediators. Gastroenterology 2000, 118, 1001-1011.

22. Ogawa, A.; Andoh, A.; Araki, Y.; Bamba, T.; Fujiyama, Y. Neutralization of interleukin-17 aggravates dextran sulfate sodium-induced colitis in mice. Clin. Immunol. 2004, 110, 55-62.

23. Strzępa, A.; Szczepanik, M. IL-17-expressing cells as a potential therapeutic target for treatment of immunological disorders. Pharmacol. Rep. 2011, 63, 30-44.

24. O’Connor, W., Jr.; Kamanaka, M.; Booth, C.J.; Town, T.; Nakae, S.; Iwakura, Y.; Kolls, J.K.; Flavell, R.A. A protective function for interleukin 17A in T cell-mediated intestinal inflammation. Nat. Immunol. 2009, 10, 603-609.

25. Yang, X.O.; Chang, S.H.; Park, H.; Nurieva, R.; Shah, B.; Acero, L.; Wang, Y.H.; Schluns, K.S.; Broaddus, R.R.; Zhu, Z.; et al. Regulation of inflammatory responses by IL-17F. J. Exp. Med. 2008, 205, 1063-1075. 
26. Tanabe, S.; Kinuta, Y.; Saito, Y. Bifidobacterium infantis suppresses proinflammatory interleukin-17 production in murine splenocytes and dextran sodium sulfate-induced intestinal inflammation. Int. J. Mol. Med. 2008, 22, 181-185.

27. Griffin, G.K.; Newton, G.; Tarrio, M.L.; Bu, D.X.; Maganto-Garcia, E.; Azcutia, V.; Alcaide, P.; Grabie, N.; Luscinskas, F.W.; Croce, K.J.; et al. IL-17 and TNF- $\alpha$ sustain neutrophil recruitment during inflammation through synergistic effects on endothelial activation. J. Immunol. 2012, 15, 6287-6299.

28. Taylor, P.R.; Roy, S.; Leal, S.M.; Sun, Y.; Howell, S.J.; Cobb, B.A.; Li, X.; Pearlman, E. Activation of neutrophils by autocrine IL-17A-IL-17RC interactions during fungal infection is regulated by IL-6, IL-23, ROR $\gamma \mathrm{t}$ and dectin-2. Nat. Immunol. 2014, 15, 143-152.

29. Prause, O.; Miyamoto, M.; Sjöstrand, M.; Laan, M.; Lötvall, J.; Lindén, A. Endogenous IL-17 as a mediator of neutrophil recruitment caused by endotoxin exposure in mouse airways. J. Immunol. 2003, 170, 4665-4672.

30. Neurath, M.F.; Atreya, R. Molecular pathways controlling barrier function in IBD. Nat. Rev. Gastroenterol. Hepatol. 2015, 12, 67-68.

31. Hedin, C.; Whelan, K.; Lindsay, J.O. Evidence for the use of probiotics and prebiotics in inflammatory bowel disease: A review of clinical trials. Proc. Nutr. Soc. 2007, 66, 307-315.

32. Miyauchi, E.; Ogita, T.; Miyamoto, J.; Kawamoto, S.; Morita, H.; Ohno, H.; Suzuki, T.; Tanabe, S. Bifidobacterium longum alleviates dextran sulfate sodium-induced colitis by suppressing IL-17A response: Involvement of intestinal epithelial costimulatory molecules. PLOS ONE 2013, 8, e79735.

33. Ghadimi, D.; Helwig, U.; Schrezenmeir, J.; Heller, K.J.; de Vrese, M. Epigenetic imprinting by commensal probiotics inhibits the IL-23/IL-17 axis in an in vitro model of the intestinal mucosal immune system. J. Leukoc. Biol. 2012, 92, 895-911.

34. Dotan, I.; Allez, M.; Nakazawa, A.; Brimnes, J.; Schulder-Katz, M.; Mayer, L. Intestinal epithelial cells from inflammatory bowel disease patients preferentially stimulate $\mathrm{CD}^{+} \mathrm{T}$ cells to proliferate and secrete interferon- $\gamma$. Am. J. Physiol. Gastrointest. Liver Physiol. 2007, 292, G1630-G1640.

35. Dubinsky, M.C.; Wang, D.; Picornell, Y.; Wrobel, I.; Katzir, L.; Quiros, A.; Dutridge, D.; Wahbeh, G.; Silber, G.; Bahar, R.; et al. IL-23 receptor (IL-23R) gene protects against pediatric Crohn's disease. Inflamm. Bowel Dis. 2007, 13, 511-515.

36. Siakavellas, S.I.; Bamias, G. Role of the IL-23/IL-17 axis in Crohn's disease. Discov. Med. 2012, 14, 253-262.

37. Bogaert, S.; Lauens, D.; Peeters, H.; Melis, L.; Olievier1, K.; Boon, N.; Verbruggen, G.; Vandesompele, J.; Elewaut, D.; de Vos, M. Differential mucosal expression of Th17-related genes between the inflamed colon and ileum patients with inflammatory bowel disease. BMC Immunol. 2010, 11, 1-12.

38. Seiderer, J.; Elben, I.; Diegelmann, J.; Glas, J.; Stallhofer, J.; Tillack, C.; Pfennig, S.; Jürgens, M.; Schmechel, S.; Konrad, A.; et al. Role of the novel Th17 cytokine IL-17F in inflammatory bowel disease (IBD): Upregulated colonic IL-17F expression in active Crohn's disease and analysis of the IL17F p.His161Arg polymorphism in IBD. Inflamm. Bowel Dis. 2008, 14, 437-445.

39. Takahashi, N.; Vereecke, L.; Bertrand, M.J.; Duprez, L.; Berger, S.B.; Divert, T.; Gonçalves, A.; Sze, M.; Gilbert, B.; Kourula, S.; et al. RIPK1 ensures intestinal homeostasis by protecting the epithelium against apoptosis. Nature 2014, 513, 95-99. 
40. Sanders, M.E.; Guarner, F.; Guerrant, R.; Holt, P.R.; Quigley, E.M.; Sartor, R.B.; Sherman, P.M.; Mayer, E.A. An update on the use and investigation of probiotics in health and disease. Gut 2013, 62, 787-796.

41. Spiller, R. Review article: Probiotics and prebiotics in irritable bowel syndrome. Aliment. Pharmacol. Ther. 2008, 28, 385-396.

42. Dupaul-Chicoine, J.; Dagenais, M.; Saleh, M. Crosstalk between the intestinal microbiota and the innate immune system in intestinal homeostasis and inflammatory bowel disease. Inflamm. Bowel Dis. 2013, 19, 2227-2237.

43. Abraham, C.; Medzhitov, R. Interactions between the host innate immune system and microbes in inflammatory bowel disease. Gastroenterology 2011, 140, 1729-1737.

44. Bien, J.; Palagani, V.; Bozko, P. The intestinal microbiota dysbiosis and Clostridium difficile infection: Is there a relationship with inflammatory bowel disease? Ther. Adv. Gastroenterol. 2013, 6, 53-68.

45. Shanahan, F. Probiotics and inflammatory bowel disease: From fads and fantasy to facts and future. Br. J. Nutr. 2002, 88, S5-S9.

46. Lepage, P.; Häsler, R.; Spehlmann, M.E.; Rehman, A.; Zvirbliene, A.; Begun, A.; Ott, S.; Kupcinskas, L.; Doré, J.; Raedler, A.; et al. Twin study indicates loss of interaction between microbiota and mucosa of patients with ulcerative colitis. Gastroenterology 2011, 141, 227-236.

47. Takaishi, H.; Matsuki, T.; Nakazawa, A.; Takada, T.; Kado, S.; Asahara, T.; Kamada, N.; Sakuraba, A.; Yajima, T.; Higuchi, H.; et al. Imbalance in intestinal microflora constitution could be involved in the pathogenesis of inflammatory bowel disease. Int. J. Med. Microbiol. 2008, 298, $463-472$.

48. Yoon, H.; Yoon, Y.-S.; Kim, M.-S.; Chung, M.J.; Yum, D.Y. A probiotic preparation duolac-gold ameliorates dextran sulphate sodium-induced mouse colitis by downregulating the expression of IL-6. Toxicol. Res. 2014, 30, 27-32.

49. Heuvelin, E.; Lebreton, C.; Grangette, C.; Pot, B.; Cerf-Bensussan, N.; Heyman, M. Mechanisms involved in alleviation of intestinal inflammation by Bifidobacterium breve soluble factors. PLoS ONE 2009, 4, e5184.

50. Peran, L.; Camuesco, D.; Comalada, M.; Nieto, A.; Concha, A.; Adrio, J.L.; Olivares, M.; Xaus, J.; Zarzuelo, A.; Galvez, J. Lactobacillus fermentum, a probiotic capable to release glutathione, prevents colonic inflammation in the TNBS model of rat colitis. Int. J. Colorectal Dis. 2006, 21, 737-746.

51. Damaskos, D.; Kolios, G. Probiotics and prebiotics in inflammatory bowel disease: Microflora "on the scope". Br. J. Clin. Pharmacol. 2008, 65, 453-467.

52. Ewaschuk, J.B.; Dieleman, L.A. Probiotics and prebiotics in chronic inflammatory bowel diseases. World J. Gastroenterol. 2006, 12, 5941-5950.

53. Seksik, P.; Dray, X.; Sokol, H.; Marteau, P. Is there any place for alimentary probiotics, prebiotics or synbiotics, for patients with inflammatory bowel disease? Mol. Nutr. Food Res. 2008, 52, 906-912.

54. Ford, A.C.; Quigley, E.M.; Lacy, B.E.; Lembo, A.J.; Saito, Y.A.; Schiller, L.R.; Soffer, E.E.; Spiegel, B.M.; Moayyedi, P. Efficacy of prebiotics, probiotics, and synbiotics in irritable bowel syndrome and chronic idiopathic constipation: Systematic review and meta-analysis. Am. J. Gastroenterol. 2014, 1547-1561. 
55. Zocco, M.; Dal Verme, L.Z.; Cremonini, F.; Piscaglia, A.C.; Nista, E.C.; Candelli, M.; Novi, M.; Rigante, D.; Cazzato, I.A.; Ojetti, V.; et al. Efficacy of Lactobacillus GG in maintaining remission of ulcerative colitis. Aliment. Pharmacol. Ther. 2006, 23, 1567-1574.

56. Ishikawa, H.; Akedo, I.; Umesaki, Y.; Tanaka, R.; Imaoka, A.; Otani, T. Randomized controlled trial of the effect of bifidobacteria-fermented milk on ulcerative colitis. J. Am. Coll. Nutr. 2003, $22,56-63$.

57. Bibiloni, R.; Fedorak, R.N.; Tannock, G.W.; Madsen, K.L.; Gionchetti, P.; Campieri, M.; de Simone, C.; Sartor, R.B. VSL\#3 Probiotic-mixture induces remission in patients with active ulcerative colitis. Am. J. Gastroenterol. 2005, 100, 1539-1546.

58. Rembacken, B.; Snelling, A.; Hawkey, P.; Chalmers, D.M.; Axon, A.T. Non-pathogenic Escherichia coli versus mesalazine for the treatment of ulcerative colitis: A randomised trial. Lancet 1999, 354, 635-639.

59. Campieri, M.; Rizzello, F.; Venturi, A.; Poggioli, G.; Ugolini, F. Combination of antibiotic and probiotic treatment is efficacious in prophylaxis of post-operative recurrence of Crohn's disease: A randomized controlled study vs mesalamine. Gastroenterology 2000, 118, A178.

60. Guslandi, M.; Mezzi, G.; Sorghi, M.; Testoni, P.A. Saccharomyces boulardii in maintenance treatment of Crohn's disease. Dig. Dis. Sci. 2000, 45, 1462-1464.

61. Prantera, C.; Scribano, M.; Falasco, G.; Andreoli, A.; Luzi, C. Ineffectiveness of probiotics in preventing recurrence after curative resection for Crohn's disease: A randomised controlled trial with Lactobacillus GG. Gut 2002, 51, 405-409.

62. Schultz, M.; Timmer, A.; Herfarth, H.H.; Sartor, R.B.; Vanderhoof, J.A.; Rath, H.C. Lactobacillus GG in inducing and maintaining remission of Crohn's disease. BMC Gastroenterol. 2004, 4, 5.

63. Bousvaros, A.; Guandalini, S.; Baldassano, R.N.; Botelho, C.; Evans, J.; Ferry, G.D.; Goldin, B.; Hartigan, L.; Kugathasan, S.; Levy, J.; et al. A randomized, double-blind trial of Lactobacillus GG versus placebo in addition to standard maintenance therapy for children with Crohn's disease. Inflamm. Bowel Dis. 2005, 11, 833-839.

64. Gupta, P.; Andrew, H.; Kirschner, B.S.; Guandalini, S. Is Lactobacillus GG helpful in children with Crohn's disease? Results of a preliminary, open-label study. J. Pediatr. Gastroenterol. Nutr. 2000, 31, 453-457.

65. Marteau, P.; Lémann, M.; Seksik, P.; Laharie, D.; Colombel, J.F.; Bouhnik, Y.; Cadiot, G.; Soulé, J.C.; Bourreille, A.; Metman, E.; et al. Ineffectiveness of Lactobacillus johnsonii LA1 for prophylaxis of postoperative recurrence in Crohn's disease: A randomised, double blind, placebo controlled GETAID trial. Gut 2006, 55, 842-847.

66. Van Gossum, A.; Dewit, O.; Louis, E.; de Hertogh, G.; Baert, F.; Fontaine, F.; DeVos, M.; Enslen, M.; Paintin, M.; Franchimont, D. Multicenter randomized-controlled clinical trial of probiotics (Lactobacillus johnsonii, LA1) on early endoscopic recurrence of Crohn's disease after ileo-caecal resection. Inflamm. Bowel Dis. 2007, 13, 135-142.

67. Gionchetti, P.; Rizzello, F.; Helwig, U.; Venturi, A.; Lammers, K.M.; Brigidi, P.; Vitali, B.; Poggioli, G.; Miglioli, M.; Campieri, M. Prophylaxis of pouchitis onset with probiotic therapy: A double-blind, placebo-controlled trial. Gastroenterology 2003, 124, 1202-1209. 
68. Mimura, T.; Rizzello, F.; Helwig, U.; Poggioli, G.; Schreiber, S.; Talbot, I.C.; Nicholls, R.J.; Gionchetti, P.; Campieri, M.; Kamm, M.A. Once daily high dose probiotic therapy (VSL\#3) for maintaining remission in recurrent or refractory pouchitis. Gut 2004, 53, 108-114.

69. Gosselink, M.P.; Schouten, W.R.; van Lieshout, L.M.; Hop, W.C.; Laman, J.D.; van Embden, J.G. Delay of the first onset of pouchitis by oral intake of the probiotic strain Lactobacillus rhamnosus GG. Dis. Colon Rectum. 2004, 47, 876-884.

70. Shen, B.; Brzezinski, A.; Fazio, V.W.; Remzi, F.H.; Achkar, J.P.; Bennett, A.E.; Sherman, K.; Lashner, B.A. Maintenance therapy with a probiotic in antibiotic-dependent pouchitis: Experience in clinical practice. Aliment. Pharmacol. Ther. 2005, 22, 721-728.

71. Kuisma, J.; Mentula, S.; Jarvinen, H.; Kahri, A.; Saxelin, M.; Farkkila, M. Effect of Lactobacillus rhamnosus GG on ileal pouch inflammation and microbial flora. Aliment. Pharmacol. Ther. 2003, 17, 509-515.

72. Geier, M.S.; Butler, R.N.; Giffard, P.M.; Howarth, G.S. Lactobacillus fermentum BR11, a potential new probiotic, alleviates symptoms of colitis induced by dextran sulfate sodium (DSS) in rats. Int. J. Food Microbiol. 2007, 114, 267-274.

73. Zoumpopoulou, G.; Foligne, B.; Christodoulou, K.; Grangetteb, C.; Potb, B.; Tsakalidou E. Lactobacillus fermentum ACA-DC 179 displays probiotic potential in vitro and protects against trinitrobenzene sulfonic acid (TNBS)-induced colitis and Salmonella infection in murine models. Int. J. Food Microbiol. 2008, 121, 18-26.

74. Mañé, J.; Lorén, V.; Pedrosa, E.; Ojanguren, I.; Xaus, J.; Cabré, E.; Domènech, E.; Gassull, M.A. Lactobacillus fermentum CECT 5716 prevents and reverts intestinal damage on TNBS-induced colitis in mice. Inflamm. Bowel Dis. 2009, 15, 1155-1163.

75. Chen, L.-L.; Zou, Y.-Y.; Lu, F.-G.; Li, F.J.; Lian, G.H. Efficacy profiles for different concentrations of Lactobacillus acidophilus in experimental colitis. World J. Gastroenterol. 2013, 19, 5347-5356.

76. Sans, M. Probiotics for inflammatory bowel disease: A critical appraisal. Dig. Dis. 2009, 27, 111-114.

77. Rolhion, N.; Darfeuille-Michaud, A. Adherent-invasive Escherichia coli in inflammatory bowel disease. Inflamm. Bowel Dis. 2007, 13, 1277-1283.

78. Bartlett, J.G. Clostridium difficile: Old and new observations. J. Clin. Gastroenterol. 2007, 41, S24-S29.

79. Venturi, A.; Gionchetti, P.; Rizzello, F.; Johansson, R.; Zucconi, E.; Brigidi, P.; Matteuzzi, D.; Campieri, M. Impact on the composition of the faecal ora by a new probiotic preparation: Preliminary data on maintenance treatment of patients with ulcerative colitis. Aliment. Pharmacol. Ther. 1999, 13, 1103-1108.

80. Venkatraman, A.; Ramakrishna, B.; Pulimood, A.B.; Patra, S.; Murthy, S. Increased permeability in dextran sulphate colitis in rats: Time course of development and effect of butyrate. Scand. J. Gastroenterol. 2000, 35, 1053-1059.

81. Claes, I.J.; de Keersmaecker, S.C.; Vanderleyden, J.; Lebeer, S. Lessons from probiotic-Host interaction studies in murine models of experimental colitis. Mol. Nutr. Food Res. 2011, 55, $1441-1453$. 
82. Ogita, T.; Tanii, Y.; Morita, H.; Suzuki, T.; Tanabe, S. Suppression of Th17 response by Streptococcus thermophilus ST28 through induction of IFN- $\gamma$. Int. J. Mol. Med. 2011, 28, 817-822.

83. Fitzpatrick, L.R.; Deml, L.; Hofmann, C.; Small, J.S.; Groeppel, M.; Hamm, S.; Lemstra, S.; Leban, J.; Ammendola, A. 4SC-101, a novel immunosuppressive drug, inhibits IL-17 and attenuates colitis in two murine models of inflammatory bowel disease. Inflamm. Bowel Dis. 2010, 16, 1763-1777.

84. Fitzpatrick, L.R.; Small, J.S.; Doblhofer, R.; Ammendola, A. Vidofludimus inhibits colonic interleukin-17 and improves hapten-induced colitis in rats by a unique dual mode of action. J. Pharmacol. Exp. Ther. 2012, 342, 850-860.

85. Iwakura, Y.; Ishigame, H. The IL-23/IL-17 axis in inflammation. J. Clin. Investig. 2006, 116, 1218-1222.

86. Mangan, P.R.; Harrington, L.E.; O’Quinn, D.B.; Helms, W.S.; Bullard, D.C.; Elson, C.O.; Hatton, R.D.; Wahl, S.M.; Schoeb, T.R.; Weaver, C.T. Transforming growth factor- $\beta$ induces development of the Th17 lineage. Nature 2006, 441, 231-234.

87. Kumar, N.; Navalpur, S.; Balamurugan, R.; Pulimood, A.; Pugazhendhi, S.; Ramakrishna, B.S. Probiotic administration alters the gut flora and attenuates colitis in mice administered dextran sodium sulfate. J. Gastroenterol. Hepatol. 2008, 23, 1834-1839.

88. Mach, T. Clinical usefulness of probiotics. J. Physiol. Pharmacol. 2006, 57, 23-33.

89. Ueno, N.; Fujiya, M.; Segawa, S.; Nata, T.; Moriichi, K.; Tanabe, H.; Mizukami, Y.; Kobayashi, N.; Ito, K.; Kohgo, Y. Heat-killed body of Lactobacillus brevis SBC8803 ameliorates intestinal injury in a murine model of colitis by enhancing the intestinal barrier function. Inflamm. Bowel Dis. 2011, $17,2235-2250$.

90. Lee, H.-S.; Han, S.-Y.; Bae, E.-A.; Huh, C.S.; Ahn, Y.T.; Lee, J.H.; Kim, D.H. Lactic acid bacteria inhibit proinflammatory cytokine expression and bacterial glycosaminoglycan degradation activity in dextran sulfate sodium-induced colitic mice. Int. Immunopharmacol. 2008, 8, 574-580.

91. Roselli, M.; Finamore, A.; Nuccitelli, S.; Carnevali, P.; Brigidi, P.; Vitali, B.; Nobili, F.; Rami, R.; Garaguso, I.; Mengheri, E. Prevention of TNBS-induced colitis by different Lactobacillus and Bifidobacterium strains is associated with an expansion of $\gamma \delta \mathrm{T}$ and regulatory $\mathrm{T}$ cells of intestinal intraepithelial lymphocytes. Inflamm. Bowel Dis. 2009, 15, 1526-1536.

92. Peran, L.; Sierra, S.; Comalada, M.; Lara-Villoslada, F.; Bailón, E.; Nieto, A.; Concha, A.; Olivares, M.; Zarzuelo, A.; Xaus, J.; et al. A comparative study of the preventative effects exerted by two probiotics, Lactobacillus reuteri and Lactobacillus fermentum, in the trinitrobenzenesulfonic acid model of rat colitis. Br. J. Nutr. 2007, 97, 96-103.

93. Peran, L.; Camuesco, D.; Comalada, M.; Nieto, A.; Concha, A.; Diaz-Ropero, M.P.; Olivares, M.; Xaus, J.; Zarzuelo, A.; Galvez, J. Preventative effects of a probiotic, Lactobacillus salivarius ssp. salivarius, in the TNBS model of rat colitis. World J. Gastroenterol. 2005, 11, 5185-5192.

94. Gad, M.; Ravn, P.; Søborg, D.A.; Lund-Jensen, K.; Ouwehand, A.C.; Jensen, S.S. Regulation of the IL-10/IL-12 axis in human dendritic cells with probiotic bacteria. FEMS Immunol. Med. Microbiol. 2011, 63, 93-107.

95. Shida, K.; Nanno, M.; Nagata, S. Flexible cytokine production by macrophages and T cells in response to probiotic bacteria: A possible mechanism by which probiotics exert multifunctional immune regulatory activities. Gut Microbes 2011, 2, 109-114. 
96. Latvala, S.; Miettinen, M.; Kekkonen, R. A.; Korpela, R.; Julkunen, I. Lactobacillus rhamnosus GG and Streptococcus thermophilus induce suppressor of cytokine signalling 3 (SOCS3) gene expression directly and indirectly via interleukin-10 in human primary macrophages. Clin. Exp. Immunol. 2011, 165, 94-103.

97. Hart, A.L.; Lammers, K.; Brigidi, P.; Vitali, B.; Rizzello, F.; Gionchetti, P.; Campieri, M.; Kamm, M.A.; Knight, S.C.; Stagg, A.J. Modulation of human dendritic cell phenotype and function by probiotic bacteria. Gut 2004, 53, 1602-1609.

98. You, J.; Yaqoob, P. Evidence of immunomodulatory effects of a novel probiotic, Bifidobacterium longum bv. infantis CCUG 52486. FEMS Immunol. Med. Microbiol. 2012, 66, 353-362.

99. Chen, R.B.Y. Insights from mouse models of colitis, J. Leukoc. Biol. 2000, 67, 267-278.

100. Annunziato, S.R.F. Do studies in humans better depict Th17 cells? Blood 2009, 114, 2213-2219.

101. Hershberg, R.M.; Mayer, L.F. Antigen processing and presentation by intestinal epithelial cells-Polarity and complexity. Immunol. Today 2000, 21, 123-128.

102. Westendorf, A.M.; Fleissner, D.; Groebe, L.; Jung, S.; Gruber, A.D.; Hansen, W.; Buer. J. CD4 ${ }^{+}$ Foxp $3^{+}$regulatory $\mathrm{T}$ cell expansion induced by antigen-driven interaction with intestinal epithelial cells independent of local dendritic cells. Gut 2009, 58, 211-219.

103. Borcherding, F.; Nitschke, M.; Hundorfean, G.; Rupp, J.; von Smolinski, D.; Bieber, K.; van Kooten, C.; Lehnert, H.; Fellermann, K.; Büning, J. The CD40-CD40L pathway contributes to the proinflammatory function of intestinal epithelial cells in inflammatory bowel disease. Am. J. Pathol. 2010, 176, 1816-1827.

104. Ivanov, I.I.; McKenzie, B.S.; Zhou, L.; Tadokoro1, C.E.; Lepelley, A.; Lafaille, J.J.; Cua, D.J.; Littman, D.R. The orphan nuclear receptor ROR $\gamma$ t directs the differentiation program of proinflammatory IL-17 $7^{+} \mathrm{T}$ helper cells. Cell 2006, 126, 1121-1133.

105. Yang, X.O.; Panopoulos, A.D.; Nurieva, R.; Chang, S.H.; Wang, D.; Watowich, S.S.; Dong, C. STAT3 regulates cytokine-mediated generation of inflammatory helper T cells. J. Biol. Chem. 2007, 282, 9358-9363.

106. Ghadimi, D.; de Vrese, M.; Heller, K.J.; Schrezenmeir, J. Effect of natural commensal-origin DNA on toll-like receptor 9 (TLR9) signaling cascade, chemokine IL-8 expression, and barrier integritiy of polarized intestinal epithelial cells. Inflamm. Bowel Dis. 2010, 16, 410-427.

107. Menard, S.; Candalh, C.; Bambou, J.C.; Terpend, N. Lactic acid bacteria secrete metabolites retaining anti-inflammatory properties after intestinal transport. Gut 2004, 53, 821-828.

108. Atarashi, T.T.K.; Honda, K. Induction of lamina propria Th17 cells by intestinal commensal bacteria. Vaccine 2010, 28, 8036-8038.

109. Atarashi, K.T.K.; Honda, K. Mechanism of Th17 cell differentiation in the intestinal lamina propria, Inflamm. Regen. 2009, 29, 263-269.

(C) 2015 by the authors; licensee MDPI, Basel, Switzerland. This article is an open access article distributed under the terms and conditions of the Creative Commons Attribution license (http://creativecommons.org/licenses/by/4.0/). 